\title{
The value of phase angle in Electrical Impedance Tomography breath detection
}

\author{
Davood Khodadad $^{1}$, Sven Nordebo ${ }^{1}$, Nima Seifnaraghi ${ }^{2}$, Rebecca Yerworth ${ }^{3}$, \\ Andreas D. Waldmann ${ }^{4}$, Beat Müller ${ }^{4}$, Inéz Frerichs ${ }^{5}$, Anton van Kaam ${ }^{6}$, Martijn. Miedema ${ }^{6}$ \\ and Richard Bayford ${ }^{2}$ \\ ${ }^{\mathbf{1}}$ Department of Physics and Electrical Engineering, Linnaeus University \\ ${ }^{2}$ Faculty of science and technology, Middlesex University \\ ${ }^{3}$ Department of Medical Physics and Biomedical Engineering, University College London \\ ${ }^{4}$ Swisstom AG, Schulstrasse 1, CH-7302 Landquart, Switzerland \\ ${ }^{5}$ Department of Anaesthesiology and Intensive Care Medicine, University Medical Centre Schleswig-Holstein, \\ Campus Kiel, Germany \\ ${ }^{6}$ Department of Neonatology, Emma Children's Hospital, Academic Medical Center, Amsterdam, The \\ Netherlands
}

\begin{abstract}
The objective of this paper is to report our investigation demonstrating that the phase angle information of complex impedance could be a simple indicator of a breath cycle in chest Electrical Impedance Tomography (EIT). The study used clinical neonatal EIT data. The results show that measurement of the phase angle from complex EIT data can be used as a complementary information for improving the conventional breath detection algorithms.
\end{abstract}

\section{INTRODUCTION}

Chest Electrical impedance tomography (EIT) is a non-invasive, non-radiative and real time dynamic monitoring tool for bedside imaging of regional electrical impedance changes induced by changes in the regional lung ventilation. Numerous studies have been published in lung function monitoring for adults [1-3] and recently for pediatric population [4-6]. However, EIT constitutes an ill-posed inverse problem. Successful EIT imaging relies therefore on the effectiveness of complementary information. For example, in chest EIT, breathing cycle information can be used for reconstructing a tidal image. In addition, in different clinical applications such as neonatal respiratory management, EIT is used for breath detection to obtain information about the exact timing of the two respiration phases: inspiration and expiration. Neonatal breath detection is still in the research phase and needs to be improved. A significant number of studies for EIT based breath detection have been published in recent years [7, 8]. The EIT complex value is used in a parametric modeling of changes during a breath cycle in [9]. However, most previous EIT studies used the real part of impedance, resistance, without paying attention to the imaginary part or reactance which eventuated in skipping phase angle information. The objective of this paper is to report our investigation demonstrating that the phase angle information of complex impedance could be a simple indicator of a breath cycle.

Section 2 of this paper describes material and methods of the study. Results of the proposed method together with a short discussion are presented in section 3 .

\section{MATERIALS AND METHODS}


The study used 20 clinical neonatal EIT data from an ongoing study within the EU-funded Continuous Regional Analysis Device for neonate Lung (CRADL) project (NCT02962505). The EIT voltage data from each neonatal patient is acquired by the Swisstom EIT system (Swisstom Landquart, Switzerland) using a textile sensor belt around the chest with 32 electrodes at the frame rate of $48 \mathrm{~Hz}$. EIT data were analyzed by calculating the sum of all voltages from 32 electrodes and a profile of relative complex impedance $(Z)$ changes generated. The time course of $Z$ during the EIT measurement was further calculated for the global lung. The complex impedance $\mathrm{Z}$ of a medium can be expressed in polar form as

$$
Z=|Z| e^{j \phi}
$$

where $j$ is the imaginary unit, $|Z|$ is complex impedance magnitude, i.e. the ratio of the measured voltage and the applied current during EIT measurement and $\phi$ is the phase angle which expresses the phase shift between current and voltage; see Fig. 1. Complex impedance $Z$ can also be dissected into real part or resistance, $\mathrm{R}=|\mathrm{Z}| \times \cos \phi$, and imaginary part or reactance, $\mathrm{X}=|\mathrm{Z}| \times \sin \phi$ in Cartesian form as

$$
Z=R+j X
$$

The phase angle $\phi$ was then calculated as

$$
\phi=\arctan (X / R) \times 180^{\circ} / \pi
$$

which is used in different imaging techniques to provide higher quality of measurements [10-12].

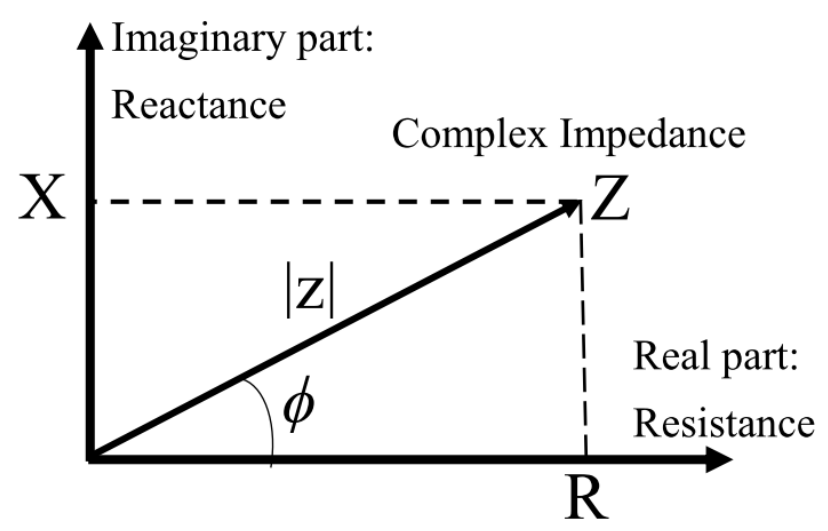

Fig. 1. A graphical representation of the complex impedance $\mathrm{Z}$ in terms of the real $\mathrm{R}$ and the imaginary $\mathrm{X}$ components and the phase angle $\phi$.

\section{RESULTS AND DISCUSSIONS}

The conventional zero crossing algorithm described in $[8,13]$ is used to detect breaths in EIT real data. Fig. 2 illustrates the corresponding breathing signal in blue color which is the input for the breath detection. The lower red (minima) and upper green (maxima) diamonds indicate the timing of endexpiration and end-inspiration, respectively. Eq. (3) is also used to calculate the phase angle $\phi$ in the complex EIT breath signal. Note that Eq. 3 results in the wrapped phase angle which is unwrapped, integrated and plotted in black color in Fig. 2. However, the plot of the unwrapped phase angle $\phi$ shows jumps of $\pm 180^{\circ}$. Significant correlation was found between the occurrence of jump in measured phase angle $\phi$ and zero cross as an indicator of a breath cycle in the real valued breath signal in the conventional breath detection method [8].The phase angle had a $\pm 180^{\circ}$ jump when there is a zero cross 
in real valued EIT breath signal. Consequently, each pair of $\pm 180^{\circ}$ jump in the measured phase angle indicates one breath cycle (Fig 2).

The results show that measurement of the phase angle $\phi$ from complex EIT data can be a great asset which contains information of breath cycles. This complementary information is potentially useful for improving the conventional breath detection algorithms, particularly during periods of irregular breathing, and hence clinical evaluations in connection with lung EIT for respiratory management and monitoring in neonatal intensive care units.

This study is an ongoing research and the next planned step is validation and optimization study by considering both impedance and complementary phase information to compare with a suitable gold standard.

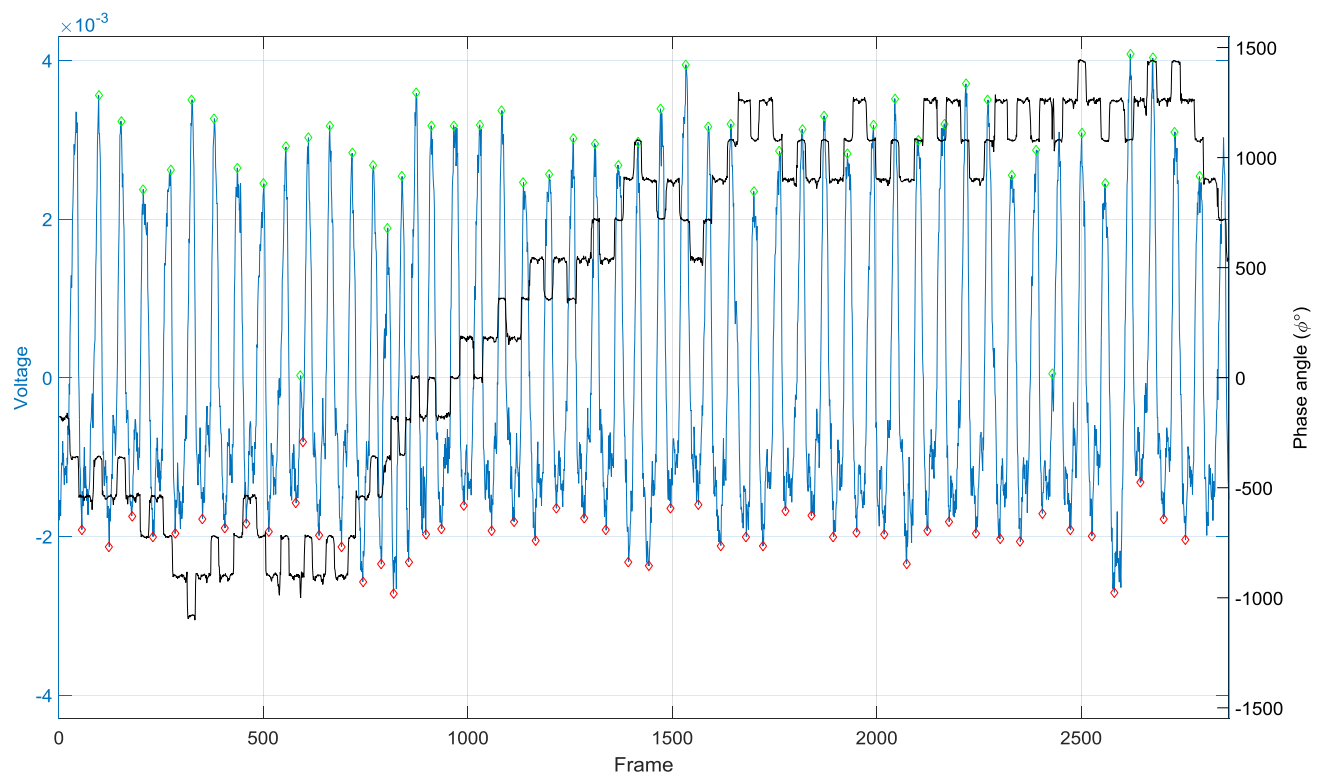

Fig. 2. Jump of $\pm 180^{\circ}$ in the measured phase angle as an indicator of zero cross in each breath cycle. The red and green diamonds indicate the detected minima and maxima during the expiration and inspiration phases, respectively, in the conventional breath detection method [8] using EIT real values.

\section{ACKNOWLEDGEMENT}

This project has received funding from the European Union's Horizon 2020 research and innovation program under grant agreement No. 668259.

\section{REFERENCES}

[1] N. Kerrouche, C. McLeod, and W. Lionheart, "Time series of EIT chest images using singular value decomposition and Fourier transform," Physiological Measurement, vol. 22, p. 147, 2001.

[2] C. A. Grant, T. Pham, J. Hough, T. Riedel, C. Stocker, and A. Schibler, "Measurement of ventilation and cardiac related impedance changes with electrical impedance tomography," Critical Care, vol. 15, p. R37, 2011.

[3] A. Adler, M. B. Amato, J. H. Arnold, R. Bayford, M. Bodenstein, and S. H. Böhm, "Whither lung EIT: where are we, where do we want to go and what do we need to get there?," Physiol Meas, vol. 33, 2012. 
[4] I. Chatziioannidis, T. Samaras, and N. Nikolaidis, "Electrical Impedance Tomography: a new study method for neonatal Respiratory Distress Syndrome?," Hippokratia, vol. 15, pp. 211-215, Jul-Sep 2011.

[5] W. Durlak and P. Kwinta, "Role of Electrical Impedance Tomography in Clinical Practice in Pediatric Respiratory Medicine," ISRN Pediatrics, vol. 2013, p. 5, 2013.

[6] F. de Souza Rossi, A. C. Z. Yagui, L. B. Haddad, A. D. A. Deutsch, and C. M. Rebello, "Electrical impedance tomography to evaluate air distribution prior to extubation in very-lowbirth-weight infants: a feasibility study," Clinics, vol. 68, pp. 345-350.

[7] I. Frerichs, M. B. P. Amato, A. H. van Kaam, D. G. Tingay, Z. Zhao, B. Grychtol, et al., "Chest electrical impedance tomography examination, data analysis, terminology, clinical use and recommendations: consensus statement of the TRanslational EIT developmeNt stuDy group," Thorax, 2016.

[8] D. Khodadad, S. Nordebo, B. Muller, A. Waldman, R. Yerworth, T. Becher, et al., "Optimized breath detection algorithm in Electrical Impedance Tomography," bioRxiv, 2018.

[9] S. Nordebo, M. Dalarsson, D. Khodadad ${ }^{1}$, B. Müller, A. D. Waldman, T. Becher, et al., "A parametric model for the changes in the complex valued conductivity of a lung during tidal breathing," Journal of Physics D: Applied Physics, vol. 51, p. 205401, 2018.

[10] I. Cseresnyés, K. Rajkai, T. Takács, and E. Vozáry, "Electrical impedance phase angle as an indicator of plant root stress," Biosystems Engineering, vol. 169, pp. 226-232, 2018/05/01/2018.

[11] T. Riedel, T. Richards, and A. Schibler, "The value of electrical impedance tomography in assessing the effect of body position and positive airway pressures on regional lung ventilation in spontaneously breathing subjects," Intensive Care Medicine, vol. 31, 2005.

[12] S. L. Swisher, M. C. Lin, A. Liao, E. J. Leeflang, Y. Khan, F. J. Pavinatto, et al., "Impedance sensing device enables early detection of pressure ulcers in vivo," Nature Communications, vol. 6, p. 6575, 03/17/online 2015 .

[13] D. Khodadad, S. Nordebo, N. Seifnaraghi, A. D. Waldmann, B. Müller, and R. Bayford, "Breath detection using short-time Fourier transform analysis in electrical impedance tomography," in 2017 XXXIInd General Assembly and Scientific Symposium of the International Union of Radio Science (URSI GASS), 2017, pp. 1-3. 